\title{
Severe DRESS syndrome after carbamazepine intake in a case with multiple addictions: A case report
}

\author{
DANIELA VRINCEANU ${ }^{1}$, MIHAI DUMITRU ${ }^{2}$, ADRIAN STEFAN $^{1}$, ADRIANA NEAGOS $^{3}$, \\ GABRIELA MUSAT $^{4}$ and ELENA ADRIANA NICA ${ }^{5}$
}

\begin{abstract}
${ }^{1}$ ENT Department, Bucharest Emergency University Hospital, 010271 Bucharest; ${ }^{2}$ Department of Anatomy, 'Carol Davila' University of Medicine and Pharmacy, 050474 Bucharest; ${ }^{3}$ ENT Department, Târgu Mureș University of Medicine and Pharmacy, 540139 Târgu Mureș; ${ }^{4}$ ENT Department, 'Sânta Maria' Clinical Hospital, 011172 Bucharest;

${ }^{5}$ Department of Anesthesia and ICU, Bucharest Emergency University Hospital, 020021 Bucharest, Romania
\end{abstract}

Received April 20, 2020; Accepted May 21, 2020

DOI: $10.3892 / \mathrm{etm} .2020 .8894$

\begin{abstract}
A case is presented of a male with drug rash with eosinophilia and systemic symptoms (DRESS) syndrome induced by carbamazepine intake. The patient presented all the elements of DRESS syndrome: Skin reaction, fever, enlargement of the lymph nodes, increased eosinophils and lymphocytes, with associated organ dysfunctions. The patient was admitted with acute laryngeal edema and imminence of respiratory insufficiency. The escalation of symptoms for this syndrome is typical, even after the administering of the the culprit medicine has ceased. However, in this case, the most difficult aspect was the complex treatment scheme prior to admission. All medical compounds involved in the background treatment were substituted with other substances in order to control the immune response. Current knowledge regarding DRESS is reviewed and possible influence of various etiologies over the present case are discussed. Clinicians should be aware of this rare situation with life-threatening potential. We benefited from the advantage of reuniting the knowledge of a complex team of experts from various tertiary emergency units in Romania.
\end{abstract}

\section{Introduction}

Drug rash with eosinophilia and systemic symptoms (DRESS) syndrome represents an severe adverse reaction after medication and is defined by the following elements: Skin symptoms, fever, lymph node enlargement, eosinophilia and modified lymphocytes, as well as internal organs dysfunction $(1,2)$.

Correspondence to: Dr Mihai Dumitru, Department of Anatomy, 'Carol Davila' University of Medicine and Pharmacy, 33/1 Sarandy Frosa, 050474 Bucharest, Romania

E-mail: orldumitrumihai@yahoo.com

Key words: DRESS syndrome, eosinophilia, respiratory distress, carbamazepine, addiction
This syndrome appears frequently after anticonvulsant therapy, usually after a period of up to 6 weeks following the beginning of the treatment and the possibility of worsening the symptoms after drug withdrawal $(3,4)$. In addition, there are other drugs responsible for DRESS, such as nonsteroidal anti-inflammatory compounds, antidepressants and some antimicrobial substances $(5,6)$.

The difficulty in diagnosing DRESS resides in the fact that eosinophilia is inconstant and skin and systemic symptoms are variable $(7,8)$. It is a life-threatening condition and it requires early diagnosis with immediate withdrawal of the drug, as well as hospitalization $(9,10)$.

\section{Case report}

We encountered a male patient aged 49, with a history of morbid obesity undergoing bariatric surgery and alcohol addiction. Patient informed consent for publication of the data/images associated with the manuscript was obtained. The authors followed the international and national regulations in accordance with the Declaration of Helsinki and all identifying information was removed.

The patient received chronic treatment with carbamazepine, diazepam, zolpidem and cipralex (escitalopram) for 1 month in order to treat his alcohol addiction. Upon admission, the patient presented right ear pain for 7 days, recurrent fever and satellite enlarged lymph nodes on the inferior parotid gland pole. He had self-administered amoxicillin $500 \mathrm{mg}$ every $12 \mathrm{~h}$ for 2 days, without previous allergic reactions. Subsequently, he developed a generalized skin reaction with erythema and pruriginous papules. Over the previous $24 \mathrm{~h}$, he had trouble swallowing and cough (Fig. 1).

Blood results revealed marked eosinophilia without leukocytosis: 6,010 leucocytes, $14.7 \%$ eosinophils, $125,000 / \mathrm{mm}^{3}$ platelets. In addition, he presented respiratory alkalosis, due to hyperventilation, with $\mathrm{pH}$ of $7.434,32 \mathrm{mmHg} \mathrm{pCo}_{2}$ and $53.9 \mathrm{mmHg} \mathrm{pO}_{2}$. Respiratory distress signaled with $85 \% \mathrm{O}_{2}$ saturation in breathing air and $91 \%$ with oxygen mask. Liver enzymes $\mathrm{ALT}=114 \mathrm{U} / 1, \mathrm{AST}=66 \mathrm{U} / 1$ and reactive $\mathrm{C}$ protein above $30 \mathrm{mg} / \mathrm{l}$; with coagulation status of INR 1.37 and PT $63 \%$. 


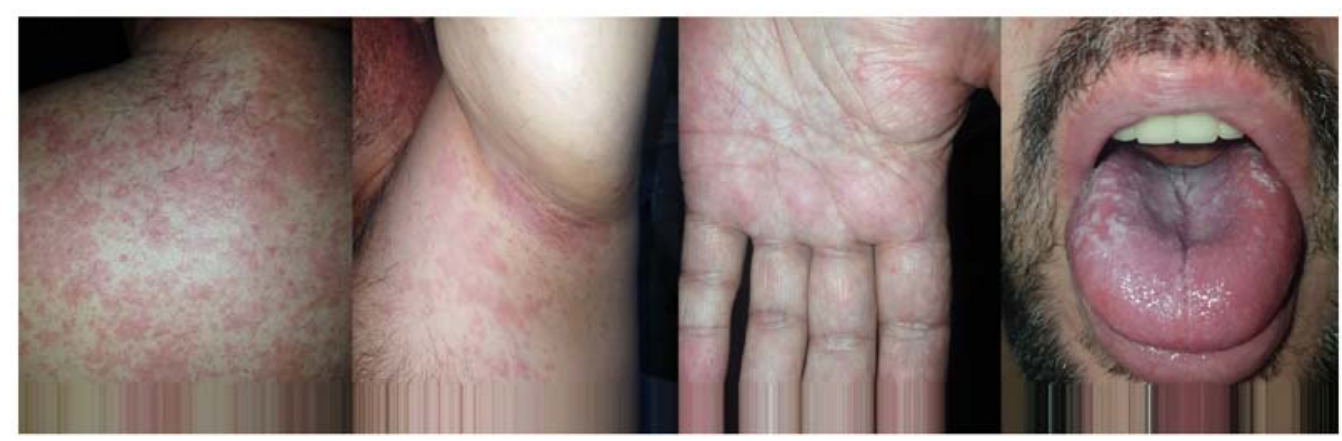

Figure 1. Case images on admission: Dorsal, axillar, palmar skin eruption without migration on the body; oral enanthem and edema with impaired breathing.

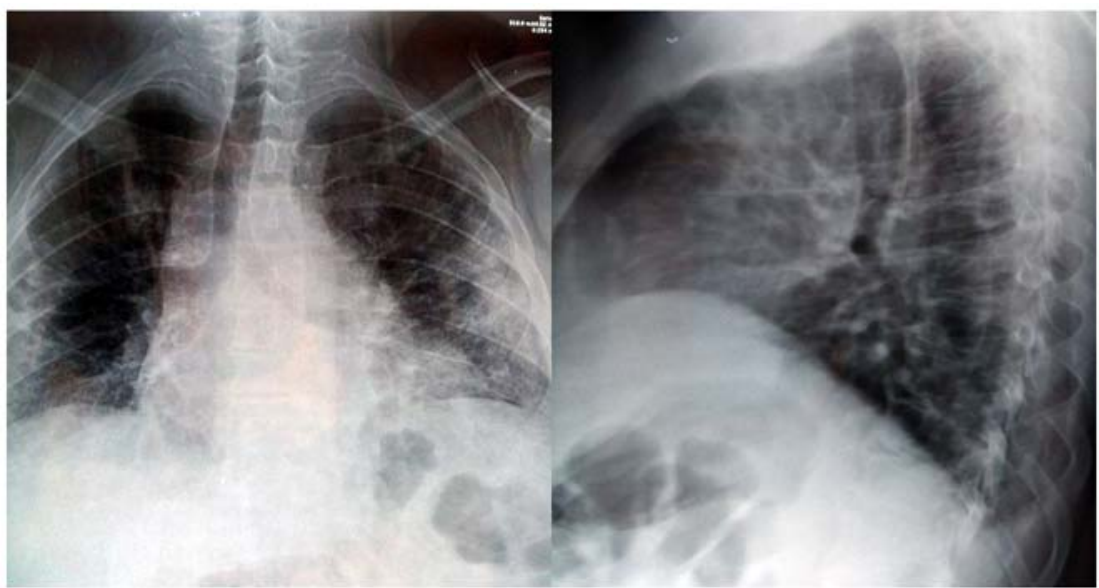

Figure 2. Chest X-ray; frontal and lateral view revealing accentuated basal interstitial accumulation of edema.

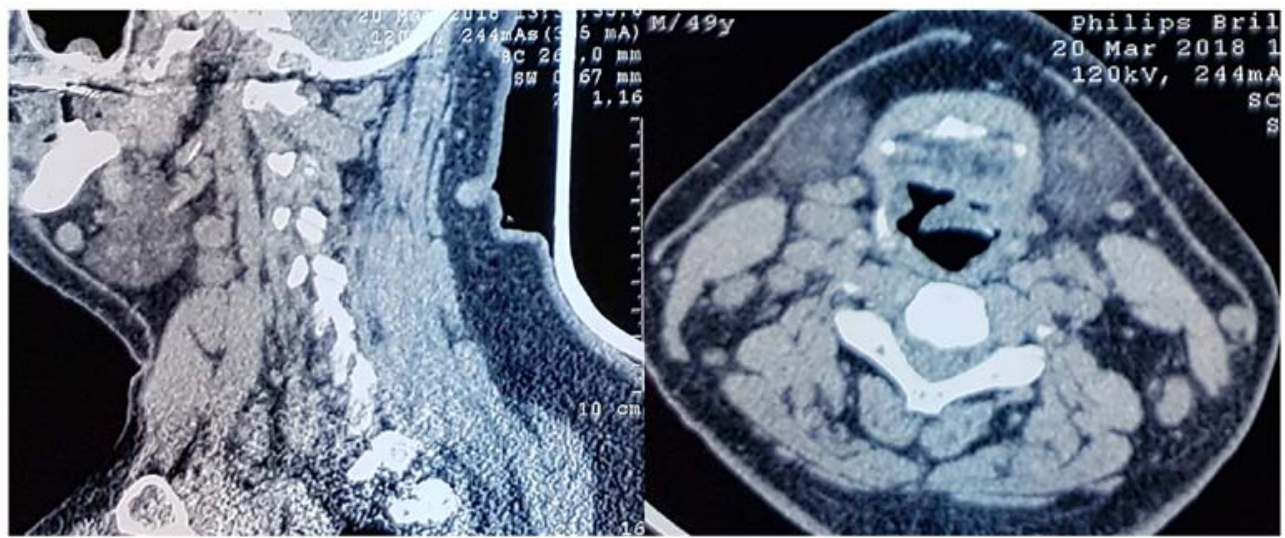

Figure 3. Neck CT scans in sagittal and axial planes, showing the presence of reactive lymph nodes and the laryngeal edema with imminent respiratory distress.

The thoracic X-ray underlined diminished transparency in both lungs, with associated alveolar opacities. In the right side, the authors observed a small transparency in the hilum, indicating a possible old cavern, as well as enlarged cardiac opacity through enlargement of the inferior left arch (Fig. 2).

On admission, the patient presented altered general status with whole body skin reaction and creasing of palms and legs. General data: Body weight $147 \mathrm{~kg}$, height $185 \mathrm{~cm}$ and body temperature 36.2 Celsius degrees.

The ENT clinical exam recorded a right parotid lymph node with pain on palpation, an edema of the epiglottis without the possibility of visualizing the vocal cords and inflammation of oral and lingual mucosa.

The patient was admitted to our ENT clinic under the suspicion of acute edema of the epiglottis due to an allergic reaction and generalized skin reaction after medication. Systemic treatment with dexamethasone at $12 \mathrm{~h}$ was provided, arnetin (ranitidine) at $12 \mathrm{~h}$, desloratadine at $12 \mathrm{~h}$, clindamycin $300 \mathrm{mg}$ at $8 \mathrm{~h}$, as indicated by the specialist of infectious diseases. The allergy examination recommended stopping the administering of carbamazepine due to a DRESS suspicion. The psychiatry examination diagnosed a background of 
depression and chronic alcohol consumption and changed the medication scheme to zolpidem once per day, diazepam $10 \mathrm{mg}$ distributed in three fractions per day, Depakine (valproic acid) $150 \mathrm{mg}$ twice per day, as well as stopping carbamazepine. The internal medicine specialist recommended the continuation of antibiotics, plus corticoid and antihistaminic medication. The case had a favorable evolution after the first $24 \mathrm{~h}$, with lowering of the eosinophils and remission of the laryngeal edema.

A thorax CT scan was performed without contrast media, which revealed interstitial densities with honeycomb aspect in the periphery without pleural reaction. However, various mediastinum lymph nodes of up to $12 \mathrm{~mm}$ in diameter were noted. This raised the suspicion of an infectious process overlying the idiopathic interstitial pneumonia (Fig. 3).

After 5 days, worsening of the skin condition was observed with a novel increase of eosinophils: up to $20.2 \%$ of 8,500 leucocytes $/ \mathrm{mm}^{3}$. Antibody levels of EBV, CMV, rubella, toxoplasma, toxocara were in the normal range. The laryngeal edema was in remission. In addition, this episode associated a fever spike of 38.1 degrees Celsius.

Taking into consideration the joint opinion of our allergy, dermatology and infectious diseases specialists, the authors referred the case to a tertiary university dermatology clinic for further treatment.

\section{Discussion}

This case reunited all the criteria for carbamazepine-induced DRESS: Acute skin reaction, fever above 38 degrees Celsius, and the presence of enlarged lymph nodes both in the neck and mediastinum, increased liver enzymes and eosinophilia (11). Moreover, there was lung involvement due to eosinophilic interstitial infiltration.

Additional criteria, such as hospital admission and the reaction triggered by medication were also present (12). The patient received carbamazepine for 3 weeks, in addition to the previous antidepressant therapy and the self-administering of penicillin-derived antibiotics, taken 1 week prior to the skin reaction.

Taking into consideration the chest $\mathrm{X}$-ray, the authors also initiated antibiotic treatment with clindamycin, which can be used even in severe cases of penicillin anaphylactic shock (13). The most difficult aspect of this case was the progression towards acute respiratory distress through acute epiglottis edema. This case also presented increased progression of symptoms, despite the suspected drug retrieval and a period of $\sim 72 \mathrm{~h}$ of systemic corticoid and antihistaminic therapy. Such late response to common treatment for controlling systemic immune response is considered extremely rare and lifethreatening (14).

This case tested negative for tuberculosis, but the authors could not ascertain the presence of HHV type 6, which has been previously associated with drug sensitivity reactions by Pereira de Silva et al (15).

The major difficulty in this case resided in the complex drug scheme self-administered by the patient prior to admission, as well as the necessity to withdraw all of these compounds and to replace them with other drugs while controlling associated pathologies and comorbidities.
In conclusion, the elements defining DRESS and the pathology mechanisms are not fully understood and any new reported case brings new data to this puzzle. The case presented unites the criteria of DRESS after carbamazepine, including additional aspects. Among the specific elements were the pulmonary interstitial reaction and the acute epiglottis edema with the prospect of acute respiratory insufficiency. This is a life-threatening situation with evolution even after the drug withdrawal. The authors consider that hospital admission is mandatory and the systemic treatment with corticoids and antihistamines is broadly approved.

\section{Acknowledgements}

Professional editing, linguistic and technical assistance was provided by Irina Radu, Individual Service Provider, certified translator in Medicine and Pharmacy (certificate credentials: Series E no. 0048).

\section{Funding}

No funding was received.

\section{Availability of data and materials}

The datasets used and/or analyzed during the current study are available from the corresponding author on reasonable request.

\section{Authors' contributions}

DV and MD treated the patient, performed literature research and wrote the manuscript. AS treated the patient and was also involved in the conception of the study. AN, GM and EAN offered second opinion during the patient treatment, performed literature research and wrote the manuscript. All authors read and approved the final manuscript.

\section{Ethics approval and consent to participate}

The study followed the international and national regulations in accordance with the Declaration of Helsinki.

\section{Patients consent for publication}

Patient informed consent for publication of the data/images associated with the manuscript was obtained. The authors followed the international and national regulations in accordance with the Declaration of Helsinki and all identifying information was removed.

\section{Competing interests}

The authors declare that they have no competing interests.

\section{References}

1. Cho YT, Yang CW and Chu CY: Drug reaction with eosinophilia and systemic symptoms (DRESS): An interplay among drugs, viruses, and immune system. Int J Mol Sci 18: 1243-1264, 2017. 
2. Wu X, Yang F, Chen S, Xiong H, Zhu Q, Gao X, Xing Q and Luo X: Clinical, viral and genetic characteristics of drug reaction with eosinophilia and systemic symptoms (DRESS) in Shanghai, China. Acta Derm Venereol 98: 401-405, 2018.

3. El Omairi N, Abourazzak S, Chaouki S, Atmani S and Hida M: Drug reaction with eosinophilia and systemic symptom (DRESS) induced by carbamazepine: A case report and literature review. Pan Afr Med J 18: 9, 2014.

4. Hiransuthikul A, Rattananupong T,Klaewsongkram J, RerknimitrP, Pongprutthipan M and Ruxrungtham K: Drug-induced hypersensitivity syndrome/drug reaction with eosinophilia and systemic symptoms (DIHS/DRESS): 11 years retrospective study in Thailand. Allergol Int 65: 432-438, 2016.

5. Musette P and Janela B: New insights into drug reaction with eosinophilia and systemic symptoms pathophysiology. Front Med (Lausanne) 4: 179, 2017.

6. Oliveira AM, Carvalho R, Martins A and Reis J: Acute hepatitis in the DRESS syndrome. GE Port J Gastroenterol 23: 304-308, 2016.

7. Wang L and Li LF: Difficult clinical management of antituberculosis DRESS syndrome complicated by MRSA infection: A case report. Medicine (Baltimore) 96: e6346, 2017.

8. Munch M, Peuvrel L, Brocard A, Saint Jean M, Khammari A, Dreno B and Quereux G: Early-onset vemurafenib-induced DRESS syndrome. Dermatology 232: 126-128, 2016.

9. Anghel AG, Anghel I, Dumitru M and Soreanu CC: Respiratory and phonatory impairment due to iatrogenic vocal fold paralysis and paresis. A retrospective study of 188 patients. Rev Med Leg 20: 287-290, 2012.
10. Thongsri T, Chularojanamontri L and Pichler WJ: Cardiac involvement in DRESS syndrome. Asian Pac J Allergy Immunol 35: 3-10, 2017.

11. Bommersbach TJ, Lapid MI, Leung JG, Cunningham JL, Rummans TA and Kung S: Management of psychotropic drug-induced DRESS syndrome: A systematic review. Mayo Clin Proc 91: 787-801, 2016.

12. Misthaq AR, Pirasath S and Sugathapala AG: DRESS syndrome associated with sulfasalazine therapy. Jaffna Med J 31: 46-47, 2019.

13. Ozdemir O and Genc G: Drug reaction with eosinophilia and systemic symptoms syndrome associated with ampicillin-sulbactam and clindamycin: A case report. Istanb Med J 20: 256-260, 2019.

14. Kang SY, Kim J, Ham J, Cho SH, Kang HR and Kim HY: Altered $\mathrm{T}$ cell and monocyte subsets in prolonged immune reconstitution inflammatory syndrome related with DRESS (drug reaction with eosinophilia and systemic symptoms). Asia Pac Allergy 10: e2, 2020.

15. Pereira de Silva N, Piquioni P, Kochen S and Saidon P: Risk factors associated with DRESS syndrome produced by aromatic and non-aromatic antipiletic drugs. Eur J Clin Pharmacol 67: 463-470, 2011 